\title{
Modelling and Parameters Identification of a Quadrotor Using a Custom Test Rig
}

\author{
Mohammad Shafiq Mohammad Ashraf', Mohamad Shukri Zainal Abidin², \\ Mohd Saiful Azimi Mahmud ${ }^{3}$, Muhammad Khairie Idham Abd Rahman', \\ Zakarya Mohammed Nasser Saleh Motea ${ }^{5}$ \\ Department of Control and Mechatronics, Faculty of Electrical Engineering, Universiti Teknologi Malaysia Skudai, \\ Malaysia
}

\begin{tabular}{l} 
Article Info \\
\hline Article history: \\
Received Dec 21 \\
Revised Jan 22 \\
Accepted Feb 7 \\
\hline Keyword: \\
Identification \\
Modelling \\
Parameters \\
Quadrotor \\
Test rig
\end{tabular}

Article Info

cle history:

\begin{abstract}
Quadrotor by nature is a very unstable system and flying it without any feedback control algorithm is deemed impossible. However, before designing the control system, system identification need to be conducted as the accuracy of the control system depends highly on the accuracy of the model. Therefore, this paper explained the design of the quadrotor model with an "X" configuration using the Euler-Newton model. Two types of test rig were designed to measure the thrust coefficient, torque coefficient and throttle command relation parameter needed in the model. Other parameter such as moment of inertia was also being measured by separating the quad rotor model into several sections: Motors, Electronics Speed Controllers (ESC) and Central Hub. All parameters needed in the designed quad rotor model has been successfully identified by measuring the parameters using the custom-built quad rotor and test rigs. The parameters found in this paper will be used in designing the control system for the quadrotor.
\end{abstract}

Copyright (C) 2018 Institute of Advanced Engineering and Science. All rights reserved.

\section{Corresponding Author:}

Mohamad Shukri Zainal Abidin,

Department of Control and Mechatronics,

Faculty of Electrical Engineering,

Universiti Teknologi Malaysia,

UTM Johor Bahru, 81310 Johor, Malaysia

Email: shukri@utm.my

\section{INTRODUCTION}

Recently, research community tend to focus on unmanned aerial vehicles (UAV) as it has a great potential and has been applied in many applications such as security surveillance, disaster management, crop monitoring, on-board imaging and educational purposes [1]-[4]. Quadrotors have become very popular due to their small size, simplicity of mechanics and good maneuverability [5]. Even though quadrotors can bring a whole lot of new application cluster, the research has been stalled until recently. The quadrotor is significantly under actuated since it only has four rotors (actuator) to completely control 6 degrees of freedoms. The degrees of freedom to be controlled are three rotational, $\operatorname{Yaw}(\dot{\psi})$, Pitch $(\dot{\theta})$ and $\operatorname{Roll}(\dot{\phi})$ and 3 translations, $X, Y, Z$.

The modeling and control system development of the quadrotor has been covered by many scholars in their work with varying complexity [6]-[8]. For instance, in [9], the author modeled the quadrotor using Newton-Euler model and the modeling includes the fundamental dynamic model of the motors, while [10] have modeled the quadrotor only for z-axis maneuver empirically and of first order only. The author of [11] also modeled the quadrotor using Newton-Euler model, however modeled the rotor dynamics empirically which makes the model hardware specific. Other scholars used Euler-Newton model to model their quadrotor dynamics also include [12], [13] and [14]. The Euler-Newton method is fundamentally accurate and is 
suitable to be used in simulation analysis. When it comes to actual implementation, the process becomes over too difficult practically but, some parameter identification can be simplified by estimating these parameters empirically as discussed by [15].

It is almost impossible to define the quadrotor parameters based on passport data components that are usually included in its composition, as the detailed information about their product rarely provided by manufacturers. In addition, the dynamic characteristics of the quadrotor system depends on the union characteristics of all its elements especially to those system that are custom built. Therefore, the special measuring test rigs [16]-[20] are used to solve this problem by providing the measurement of thrust force and the aerodynamic torque that are hard to be measured. It focused mainly on the identification parameters of fixed step propeller drives system. Therefore, test rig is a system that are required to allow quadrotor parameter measurement, as a physical plan and it is being held without linear displacement and usually have three degrees of freedom.

In this paper, a parameter identification process was conducted by measuring the value of mass moment of inertia $(\mathrm{J})$, thrust coefficient $\left(C_{T}\right)$, torque coefficient $\left(C_{Q}\right)$, throttle command relation $\left(C_{R}\right)$ and motor time constant. Two custom test rigs were built to measure the $C_{T}, C_{Q}$ and $C_{R}$ parameter based on the principle of thrust and torque coefficient. The parameter found in this paper will be used to represent a quadrotor system with an "X" configuration. The parameter identification process was conducted by taking the real quadrotor parameter measurement in the lab. The quadrotor model used in this paper will be explained in Section 2 and parameter identification process will be described in detail in Section 3.

\section{QUADROTOR MODEL}

In this paper, the configuration chosen for the quad rotor is the " $\mathrm{X}$ " configuration. By using the vector coordinate notation, the translational position and angular position of the quad rotor with respect to the Earth frame can be defined in Equation 1 and 2 respectively.

$$
\begin{aligned}
& \boldsymbol{\eta}=\left[\begin{array}{lll}
x & y & z
\end{array}\right]^{\mathrm{T}} \\
& \boldsymbol{\jmath}=\left[\begin{array}{lll}
\phi & \theta & \psi
\end{array}\right]^{\mathrm{T}}
\end{aligned}
$$

The acceleration of the center of mass of the rigid body quad rotor is based on the forces and accelerations acting on the body are determined by the velocity state equation as described in Equation 3 . Where, $\dot{v}$ is linear acceleration of the center of mass in the body frame with respect to the earth frame, $m$ is the total mass of the quad rotor, $g$ is the gravity, $C$ is the Euler Angles rotation matrix required to translate the gravity to act in the body frame, $\Omega$ is the cross-product matrix for rotational velocity required to map rotational velocity to the time derivative of the angles.

$$
\dot{\mathrm{v}}=\left(\frac{1}{m}\right) \mathrm{F}+C g-\Omega \omega_{e}=\left[\begin{array}{c}
\dot{U} \\
\dot{V} \\
\dot{W}
\end{array}\right]
$$

When considering the motion of the quad rotor, another state equation that need to be considered is the angular velocity state equation as in Equation 4. This equation is based on Euler`s equation for rigid body dynamics. This equation describes the change in roll $(\mathrm{P})$, pitch $(\mathrm{Q})$ and yaw $(\mathrm{R})$ rates caused by the inertia, angular velocity, and the moments applied by the motor-propeller system. Where $\Omega$ is the cross-product matrix for rotational velocity and $\omega_{b}$ is the rotational velocity of the quad rotor body within the body frame.

$$
\dot{\omega}=(J)^{-1}\left[M-\Omega J \omega_{b}\right]=\left[\begin{array}{c}
\dot{P} \\
\dot{Q} \\
\dot{R}
\end{array}\right]
$$

Thrust and aerodynamics applies force on the quad rotor on the positive z-axis as given by Equation 5 .

$$
F=\left[\begin{array}{c}
0 \\
0 \\
c_{T}\left(\varpi_{1}^{2}+\varpi_{2}^{2}+\varpi_{3}^{2}+\varpi_{4}^{2}\right)
\end{array}\right]
$$

The relationship matrix between the thrust and torque with motor RPM can be constructed in Equation 6, where $d$ is the length of the arm. 


$$
M=\left[\begin{array}{c}
\tau_{\phi} \\
\tau_{\theta} \\
\tau_{\psi}
\end{array}\right]=\left[\begin{array}{c}
-d S(45) c_{T} \varpi_{1}^{2}+d S(45) c_{T} \varpi_{2}^{2}+d S(45) c_{T} \varpi_{3}^{2}-d S(45) c_{T} \varpi_{4}^{2}+J_{m} Q\left(\frac{\pi}{30}\right)\left(\varpi_{1}-\varpi_{2}+\varpi_{3}-\varpi_{4}\right) \\
-d S(45) c_{T} \varpi_{1}^{2}-d S(45) c_{T} \varpi_{2}^{2}+d S(45) c_{T} \varpi_{3}^{2}+d S(45) c_{T} \varpi_{4}^{2}+J_{m} P\left(\frac{\pi}{30}\right)\left(-\varpi_{1}+\varpi_{2}-\varpi_{3}+\varpi_{4}\right) \\
-c_{Q} \varpi_{1}^{2}+c_{Q} \varpi_{2}^{2}-c_{Q} \varpi_{3}^{2}+c_{Q} \varpi_{4}^{2}
\end{array}\right]
$$

To determine the parameters, an actual hardware is needed to be built for which the parameters of the model are to be determined empirically. In terms of Microcontroller unit, Arduino DUE was used. For gyroscope and accelerometer unit, an L3G4200D and ADXL335 from Sparkfun was used. To drive the EMAX 1047 propeller, EMAX XA2212 motor was used. For ESC, Hobbysky model was used. In terms of frame, DJI F450 and Hobbyking HK-T4A was used as a remote controller and EMAX battery was used.

\section{PARAMETER IDENTIFICATION}

In this section, the equations related to the dynamic model of the quad rotor were discussed and of which the parameters to be identified from actual physical components are $J^{b}, m, c_{T}, c_{Q}, c_{R}, b$ and $t c$. These parameters need to be determined empirically and will be discussed in detail throughout this section.

\subsection{Mass Moment of Inertia, $\mathbf{J}$}

To determine the moment of inertia, the quad rotor vehicle was separated into different components. The model of each components was represented by a simplified geometric shape of constant internal density. Then, the weight of each component was measured. The moment of inertia of each component about the $x, y$, and $z$ axes of the vehicle is determined by using the parallel-axis theorem. The total moment of inertia matrix of the vehicle, is the sum the inertias for every component about each axis. The component that needs to be modelled are the motor, the ESC, arms and the central hub. The parallel axis theorem is defined in Equation 7.

$$
J_{\text {parallel-axis }}=J_{\text {COM }}+m r^{2}
$$

Where, $J_{\text {СOM }}$ is the moment of inertia of an individual component along its own axis parallel to the axis it is to be moved, $m$ is the mass of individual component and $r$ is the perpendicular distance between the parallel axes. The modeling of each individual component of quad rotor and their mass moment of inertia along corresponding axis is calculated in the next section.

\subsubsection{Motors: Solid Cylinders}

The motors were modelled as a solid cylinder to determine the mass moment of inertia for all four motors. Figure 1 shows the quadrotor structure that indicates the dimensions that need to be considered to estimate the mass moment of inertia for motors. Equation 8 shows the equation of the mass moment of inertia for $x$ and $y$ axes and Equation 9 for $z$ axes respectively.

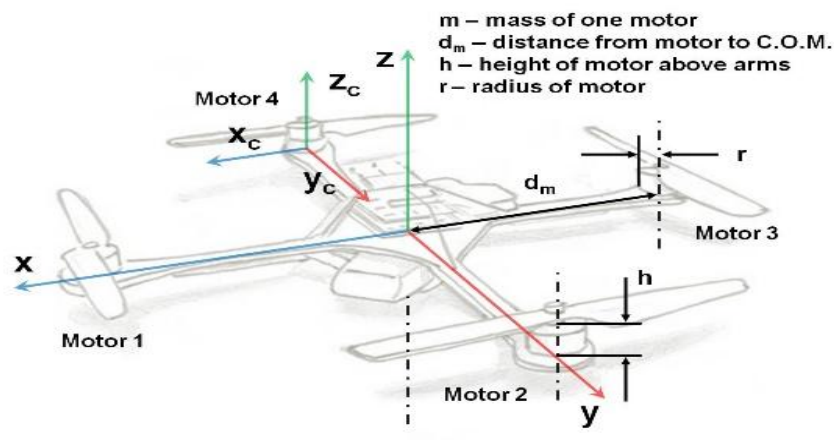

Figure 1. Dimensions to be considered for mass moment of inertia calculation of the motors

$$
\begin{aligned}
& J_{x}=J_{y}=2\left[\frac{1}{4} m r^{2}+\frac{1}{3} m h^{2}\right]+2\left[\frac{1}{4} m r^{2}+\frac{1}{3} m h^{2}+m d_{m}^{2}\right] \\
& J_{z}=4\left[\frac{1}{2} m r^{2}+m d_{m}^{2}\right]
\end{aligned}
$$


Based on the specifications that are used for the motors in this experiment, the mass $(m)$, dimensions $\left(d_{m}\right)$, height $(h)$ and radius $(r)$ was found as $m=57 \mathrm{~g}, d_{m}=26.035 \mathrm{~cm}, h=3.0 \mathrm{~cm}$ and $r=1.35 \mathrm{~cm}$. Using the dimensions measured, the moment of inertia of motor corresponding to each axis is $J_{x}=J_{y}=7.8059 \times$ $10^{-3}$ and $J_{z}=0.015475$.

\subsubsection{ESC's Thin Flat Plates}

The ESC's were modeled as thin flat plates and the mass moment of inertia for all four motors is found based on Figure 2, Equation 10 and 11. Based on the ESCs used in this project, the mass and the dimensions of the ESCs were measured to be as $\mathrm{m}=22 \mathrm{~g}$, a $=4.5 \mathrm{~cm}, \mathrm{~b}=2.6 \mathrm{~cm}, d_{s}=11.05 \mathrm{~cm}$. Using the dimensions measured, the moment of inertia of ESCs corresponding to each axis is $J_{x}=J_{y}=5.47154 \times$ $10^{-4}$ and $J_{z,}=2.17778 \times 10^{-5}$.

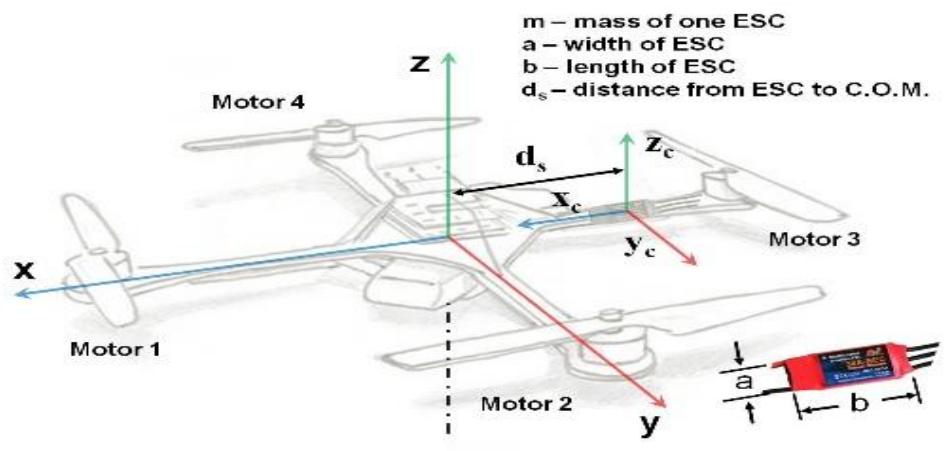

Figure 2. Dimensions to be considered for mass moment of inertia calculation of the ESCs.

$$
\begin{aligned}
& J_{x}=J_{y}=2\left[\frac{1}{12} m a^{2}\right]+2\left[\frac{1}{12} m b^{2}+m d_{s}{ }^{2}\right] \\
& J_{z}=4\left[\frac{1}{12} m\left(a^{2}+b^{2}\right)+m d_{s}{ }^{2}\right]
\end{aligned}
$$

\subsubsection{Central HUB: Solid Cylinder}

The central hub was modeled as a solid cylinder and the mass moment of inertia for the central hub was found based on Figure 3, Equations 12 and 13.

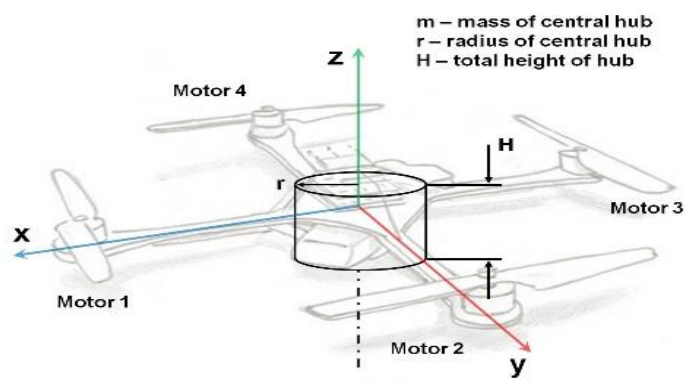

Figure 3. Dimensions to be considered for mass moment of inertia calculation of the Central HUB.

$$
\begin{aligned}
& J_{x}=J_{y}=\left[\frac{1}{4} m r^{2}+\frac{1}{12} m H^{2}\right] \\
& J_{z}=\left[\frac{1}{2} m r^{2}\right]
\end{aligned}
$$


Based on the quad rotor, the mass and the dimensions of the Central HUB were measured to be as $\mathrm{m}=335 \mathrm{~g}$, $\mathrm{r}=6.2 \mathrm{~cm}, \mathrm{H}=8.0 \mathrm{~cm}$. Using the dimensions measured, the moment of inertia of the Central HUB corresponding to each axis is as $J_{x}=J_{y}=5.006 \times 10^{-4}$ and $J_{z}=6.4387 \times 10^{-4}$.

\subsubsection{Arms: Long Cylindrical Rods}

To measure the moment of inertia for the arms, a cylindrical rods configuration was selected. The mass moment of inertia for the arms were found based on Figure 4, Equations 14 and 15.

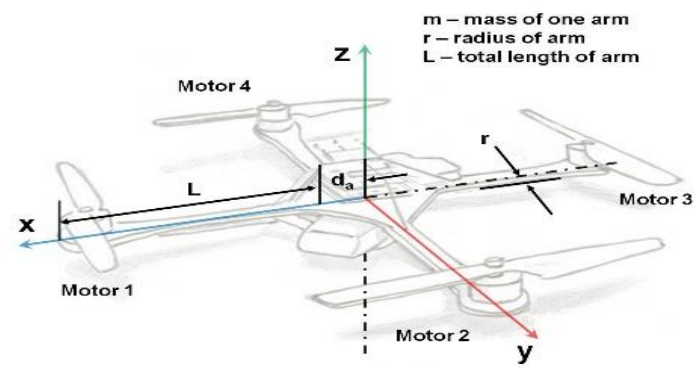

Figure 4. Dimensions to be considered for mass moment of inertia calculation of the Arms

$$
\begin{gathered}
J_{x}=J_{y}=2\left[\frac{1}{2} m r^{2}\right]+2\left[\frac{1}{4} m r^{2}+\frac{1}{3} m L^{2}+m d_{A}{ }^{2}\right] \\
J_{z}=4\left[\frac{1}{4} m r^{2}+\frac{1}{3} m L^{2}+m d_{A}{ }^{2}\right]
\end{gathered}
$$

Based on the Frame, the mass and the dimensions of the Arms were measured to be as $\mathrm{m}=57 \mathrm{~g}, \mathrm{r}=0.5 \mathrm{~cm}$, $\mathrm{L}=22 \mathrm{~cm}, d_{a}=3.5 \mathrm{~cm}$. Using the dimensions measured, the moment of inertia of the Arms corresponding to each axis is $J_{x}=J_{y}=1.98 \times 10^{-3}$ and $J_{z}=3.959 \times 10^{-3}$. Finally, by summing $J_{x}$ of all individual components, $J_{y}$ of all individual components and the $J_{z}$ of all individual components, the moment of inertia matrix is populated in Equation 16.

$$
J=\left[\begin{array}{ccc}
0.0108 & 0 & 0 \\
0 & 0.0108 & 0 \\
0 & 0 & 0.0212
\end{array}\right]
$$

\subsection{The Thrust Coefficient, $C_{T}$}

The thrust coefficient can be found by determining the gradient of the thrust versus (RPM) ${ }^{2}$ graph. The principle of measurement of thrust can be seen as in Figure 5. The mechanism is pivoted at point A and as the thrust is generated by the motor, it can be measured at point $\mathrm{B}$.

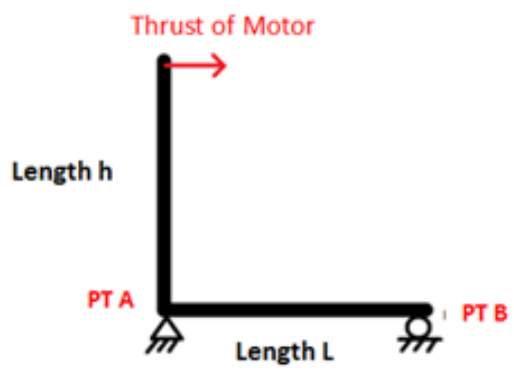

Figure 5. Principle of thrust measurement

A test rig was built to visualize the principle of measurement in Figure 5. To measure the thrust coefficient, a test rig shown in Figure 6(a) was built. To capture the RPM value, an electronics system was designed as shown in Figure 6(b). The RPM value was simply captured by using phototransistor, which detects the blade crossing, and RPM value was determined by measuring the time between the consecutive blade crossings. This data was then fed into an Arduino based microcontroller and then imported to 
Microsoft Excel to be plotted into a graph of thrust versus (RPM) ${ }^{2}$. To capture the thrust, a simple household digital weight was used.

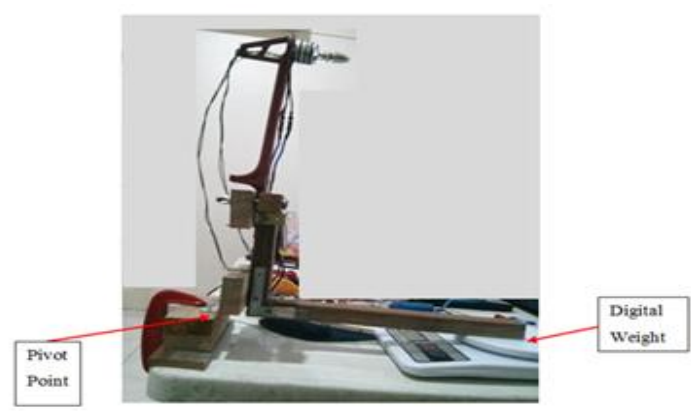

(a)

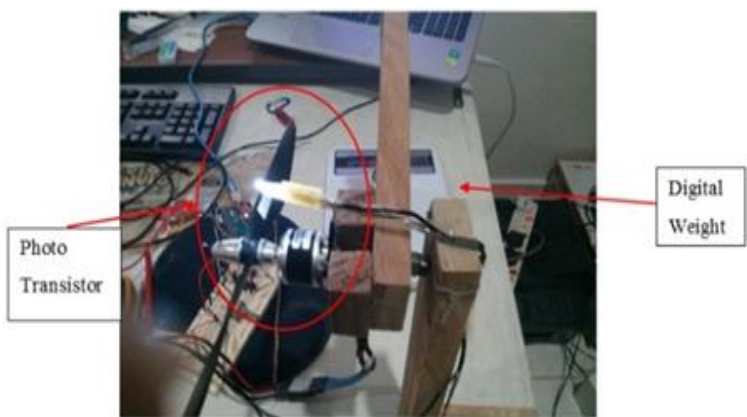

(b)

Figure 6. (a) Test rig to measure Thrust Coefficient. (b) Electronics system to measure RPM

To find the thrust coefficient, the Thrust value vs $R P M^{2}$ graph was drawn and the best-fit gradient was then determined. Figure 7 shows the data captured on the graph of thrust vs (RPM) ${ }^{2}$. Then, a linear approximation was made and finally the thrust coefficient was found to be $2^{-7}$.

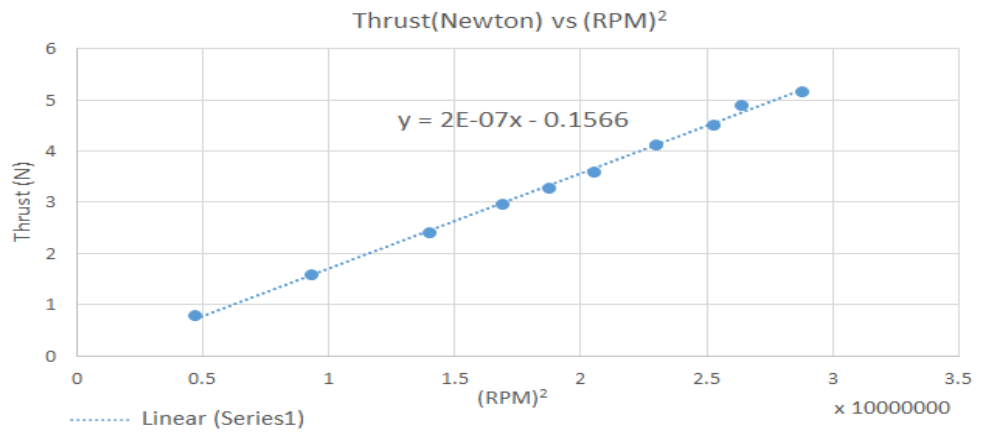

Figure 7. Graph of Newton vs (RPM) ${ }^{2}$ to determine the thrust coefficient.

\subsection{The Torque Coefficient, $C_{Q}$}

The principle of measurement of Torque Coefficient was shown in Figure 8.

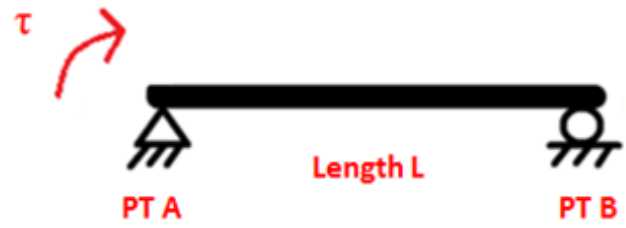

Figure 8. Principle of torque measurement

To visualize the principle of measurement in Figure 8, another test rig was built as shown in Figure 9. The torque coefficient was determined by drawing the best-fit gradient in the graph of Nm vs $R P M^{2}$. 


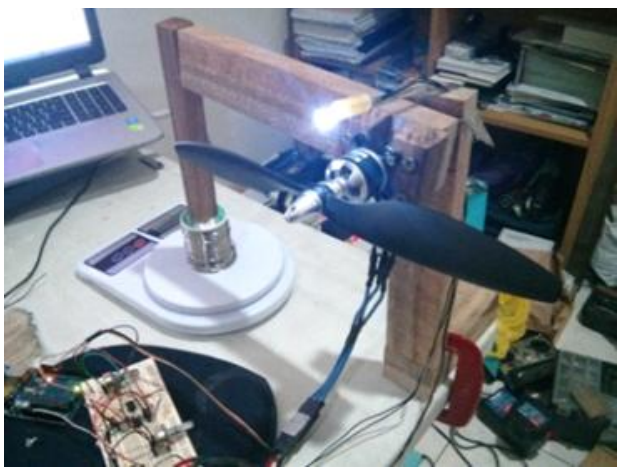

Figure 9. Test rig to measure the torque coefficient

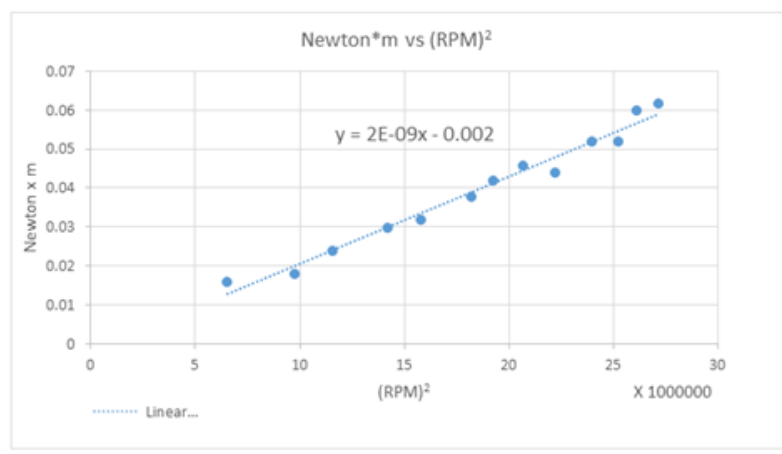

Figure 10. Graph of Newton*m vs (RPM)2 to determine the torque coefficient

Figure 10 shows the graph of torque generated by the corresponding $\mathrm{RPM}^{2}$. After performing the linear approximation, the torque coefficient was found to be $2^{-9}$.

\subsection{Throttle Command Relation, $C_{R}$}

To measure the throttle command relation, either test rig used to measure $C_{Q}$ or $C_{T}$ parameter can be used. To determine the parameter $C_{R}$ and $b$, the best-fit gradient line was drawn on the graph of RPM vs Throttle.

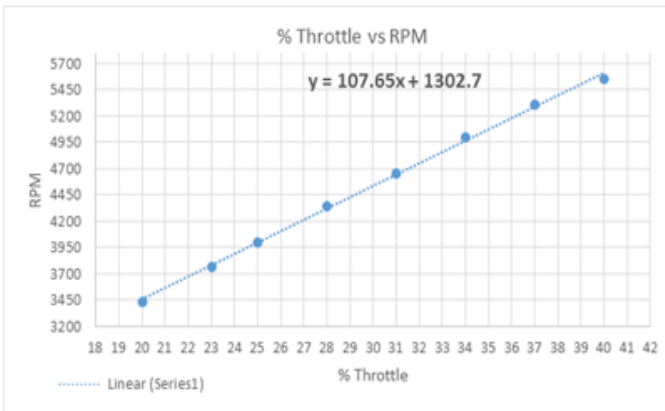

Figure 11. Graph of throttle percentage vs RPM

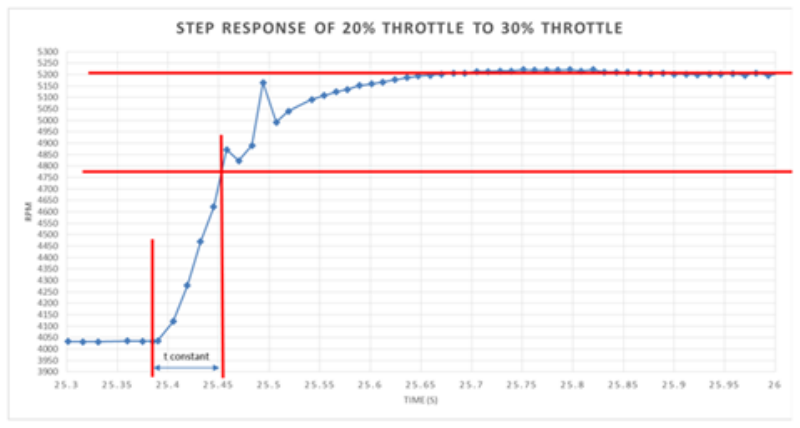

Figure $12.10 \%$ step response to determine the motor time constant

By plotting the graph of throttle percentage versus the RPM and performing the linear approximation as shown in Figure 11, $C_{R}$ was found to be 107.65 and $b$, which is the y intercept of the graph is found to be 1302.7.

\subsection{Step Input to Determine the Motor Time Constant}

The time constant of the motor is used to model the dynamics of the motor in simulation. To determine the motor constant, a step of $10 \%$ throttle is applied and the response was captured. Then, the time constant was determined at T63\% from the step response graph. Figure 12 shows the step response graph and the constant was found to be 0.07 seconds as $\mathrm{T} 0=25.385 \mathrm{~s}$ and $\mathrm{T} 63 \%=25.455 \mathrm{~s}$.

\section{CONCLUSION}

In this paper, an approach was taken to determine the parameters required for populating the mathematical model. Two custom test rigs were built by visualizing the principle of thrust and torque to measure the thrust and torque coefficient. The parameters were determined as $J_{x / y}=0.0108, J_{z}=0.0212$, $C_{T}=2^{-7}, C_{Q}=2^{-9}, C_{R}=107.65, b=1302.7$ and $t_{c}=0.07 \mathrm{~s}$. All parameters that were found in this paper will be substituted in the mathematical model for controller design in future works. The controller 
based on the parameters that were found in this paper will be designed and the simulation and experimental test will be expected to be conducted to evaluate the performance of the designed controller.

\section{ACKNOWLEDGEMENTS}

The authors are grateful to the University of Technology Malaysia and the Ministry of Higher Education (MOHE), for their partial financial support through their research funds, Vote No. R.J130000.7823.4F759.

\section{REFERENCES}

[1] B. T. M. Leong, et al., "Low-Cost Microcontroller-based Hover Control Design of a Quadcopter," Procedia Engineering, vol. 41, pp. 458-464, 2012.

[2] K. Patel and J. Barve, "Modelling, Simulation and Control Study for the Quad-Copter UAV," in Industrial and Information Systems (ICIIS) $9^{\text {th }}$ International Conference, Vols 9, pp. 1-6, 2014.

[3] Z. R. Mahayuddin et al., "Comparison of Human Pilot (Remote) Control Systems in Multirotor Unmanned Aerial Vehicle Navigation, " International Journal of Advance Science Engineering, vol. 7, pp. 132-138, 2017.

[4] J. Ajmera and V. Sankaranarayanan, "Point-to-point Control of a Quadrotor: Theory and Experiment," in IFACPapersOnLine, Vols. 49, pp. 401-406, 2016.

[5] K. Alexis, et al., "Switching Model Predictive Attitude Control for a Quadrotor Helicopter Subject to Atmospheric Disturbances, " Control Engineering Practice, vol. 19, pp. 1195-1207, 2011.

[6] C. L. Zhang and K. T. Chong, "Nonlinear Hybrid Controller for a Quadrotor Based on Sliding Mode and Backstepping," International Journal of Robotics and Automation, vol. 4, pp. 209-218, September 2015.

[7] N. M. Raharja, E. Firmansyah, A. I. Cahyadi, I., Iswanto, "Hovering Control of Quadrotor Based on Fuzzy Logic," International Journal of Power Electronics and Drive Systems, vol. 8, pp. 492-504, March 2017.

[8] A. Jabbar and F. M. Malik, " Sampled-Data Backstepping Control of a Quadrotor Unmanned Aerial Vehicle," Intenational Journal of Robotics and Automation, vol. 4, pp. 124-134, June 2015.

[9] T. Bresciani, "Modelling, Identification and Control of a Quadrotor Helicopter," Master's Thesis', Department of Automatic Control, Lund University, Sweden, 2008.

[10] J. Bazin, et al., "Feasibility of In-Flight Quadrotor Individual Motor Thrust Measurement," in AIAA Atmospheric Flight Mechanical Conference, pp. 1-12, 2016.

[11] S. Bouabdallah and R. Siegwart, "Full Control of a Quadrotor," in 2007 IEEE/RSJ International Conference on Intelligent Robotics and Systems, pp. 1-5, 2007.

[12] S. Bouabdallah, et al., "Design and Control of an Indoor Micro Quadrotor," in ICRA 2014 IEEE International Conference, Vols. 5, pp. 4393-4398, 2004.

[13] W. Zhao and T. H. Go, "Quadcopter Formation Flight Control Combining MPC and Robust Feedback Linearization," Journal of the Franklin Institute, vol. 351, pp.1335-1355, 2014.

[14] M. Elsamanty, et al., "Methodology for Identifying Quadrotor Parameters, Attitude Estimation and Control," in IEEE/ASME International Conference on Anvanced Intelligent Mechatronics, pp. 1-6, 2013.

[15] A. Chovancova, et al., "Mathematical Modelling and Parameter Identification of Quadrotor (a Survey)," Procedia Engineering, vol. 96, pp. 172-181, 2014.

[16] K. Rahnamai, "Quadrotor Drone Thrust Measurement Apparatus," in IEEE Aerospace Conference, pp. 1-6, 2016.

[17] M. R. Hossain and N. Krouglicof, "Propeller Dynamometer for Small Unmanned Aerial Vehicle," in Electrical and Computer Engineering (CCECE) 23 ${ }^{\text {rd }}$ Canadian Conference, pp. 1-6, 2010.

[18] N. Morozovsky, et al., "An Inexpensive Open Source Dynamometer for Robotics Applications," IEEE/ASME Transactions on Mechatronics, vol. 18, pp. 1855-1860, 2013.

[19] G. M. Hoffmann, et al., "Quadrotor Helicopter Flight Dynamics and Control: Theory and Experiment," in AIAA Guidance, Navigation and Control Conference and Exhibit, pp. 1-20, 2007.

[20] N. Faundes, et. al., "Different UAV Drive Train Qualification and Parameter Sets," in $32^{\text {nd }}$ Digital Avionics Systems Conference, pp. 1-15, 2013. 